for conservation globally

Threatened

通
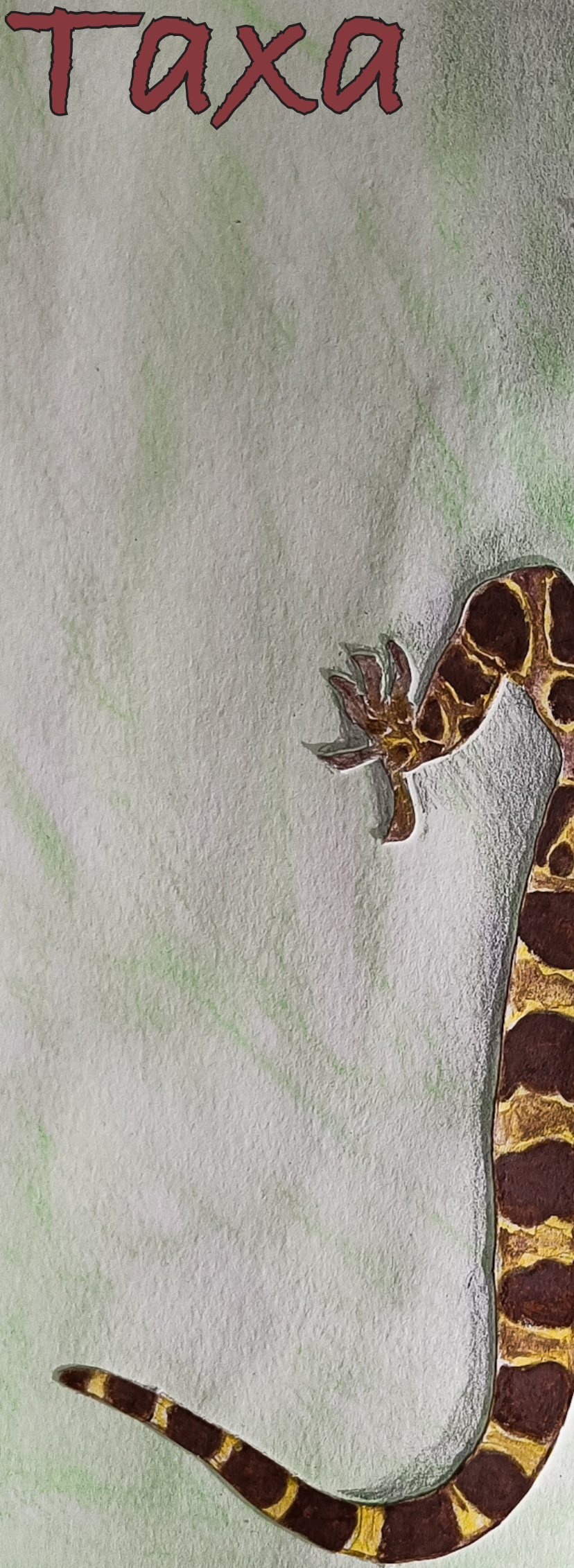

Open Access

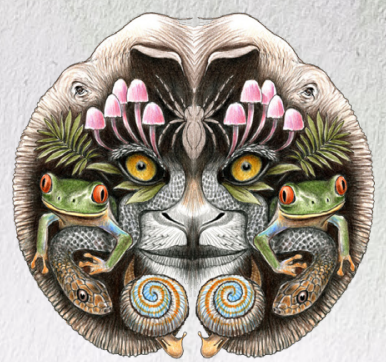

$10.1160 \mathrm{~g} / \mathrm{j}$ ott.2022.14.1.20311-20538 wWw.threatenedtaxa.org

26 January 2022 (Online \& Print) 14(1): 20311-20538 ISSN0974-7907 (Online) ISSN 0974-7893 (Print) 


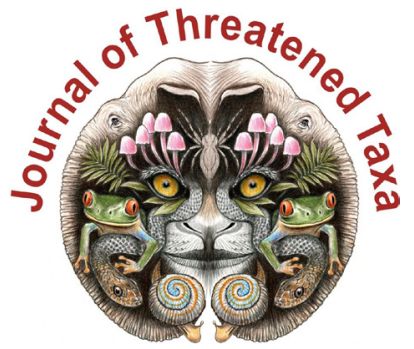

ISSN 0974-7907 (Online); ISSN $0974-7893$ (Print)

Publisher

Host

Wildlife Information Liaison Development Society

www.wild.zooreach.org

Zoo Outreach Organization www.zooreach.org

No. 12, Thiruvannamalai Nagar, Saravanampatti - Kalapatti Road, Saravanampatti, Coimbatore, Tamil Nadu 641035, India

Ph: +91 9385339863 | www.threatenedtaxa.org

Email: sanjay@threatenedtaxa.org

EDITORS

\section{Founder \& Chief Editor}

Dr. Sanjay Molur

Wildlife Information Liaison Development (WILD) Society \& Zoo Outreach Organization (ZOO),

12 Thiruvannamalai Nagar, Saravanampatti, Coimbatore, Tamil Nadu 641035, India

\section{Deputy Chief Editor}

Dr. Neelesh Dahanukar

Noida, Uttar Pradesh, India

\section{Managing Editor}

Mr. B. Ravichandran, WILD/ZOO, Coimbatore, India

\section{Associate Editors}

Dr. Mandar Paingankar, Government Science College Gadchiroli, Maharashtra 442605, India

Dr. Ulrike Streicher, Wildlife Veterinarian, Eugene, Oregon, USA

Ms. Priyanka Iyer, ZOO/WILD, Coimbatore, Tamil Nadu 641035, India

Dr. B.A. Daniel, ZOO/WILD, Coimbatore, Tamil Nadu 641035, India

\section{Editorial Board}

Dr. Russel Mittermeier

Executive Vice Chair, Conservation International, Arlington, Virginia 22202, USA

\section{Prof. Mewa Singh Ph.D., FASc, FNA, FNASc, FNAPsy}

Ramanna Fellow and Life-Long Distinguished Professor, Biopsychology Laboratory, and Institute of Excellence, University of Mysore, Mysuru, Karnataka 570006, India; Honorary Professor, Jawaharlal Nehru Centre for Advanced Scientific Research, Bangalore; and Adjunct Professor, National Institute of Advanced Studies, Bangalore

\section{Stephen D. Nash}

Scientific Illustrator, Conservation International, Dept. of Anatomical Sciences, Health Sciences Center, T-8, Room 045, Stony Brook University, Stony Brook, NY 11794-8081, USA

\section{Dr. Fred Pluthero}

Toronto, Canada

\section{Dr. Priya Davidar}

Sigur Nature Trust, Chadapatti, Mavinhalla PO, Nilgiris, Tamil Nadu 643223, India

\section{Dr. Martin Fisher}

Senior Associate Professor, Battcock Centre for Experimental Astrophysics, Cavendish

Laboratory, JJ Thomson Avenue, Cambridge CB3 OHE, UK

\section{Dr. John Fellowes}

Honorary Assistant Professor, The Kadoorie Institute, 8/F, T.T. Tsui Building, The University of Hong Kong, Pokfulam Road, Hong Kong

\section{Prof. Dr. Mirco Solé}

Universidade Estadual de Santa Cruz, Departamento de Ciências Biológicas, Vice-coordenado do Programa de Pós-Graduação em Zoologia, Rodovia Ilhéus/Itabuna, Km 16 (45662-000)

Salobrinho, Ilhéus - Bahia - Brasil

\section{Dr. Rajeev Raghavan}

Professor of Taxonomy, Kerala University of Fisheries \& Ocean Studies, Kochi, Kerala, India

\section{English Editors}

Mrs. Mira Bhojwani, Pune, India

Dr. Fred Pluthero, Toronto, Canad

Mr. P. Ilangovan, Chennai, India

Web Development

Mrs. Latha G. Ravikumar, ZOO/WILD, Coimbatore, India

\section{Typesetting}

Mr. Arul Jagadish, ZOO, Coimbatore, India

Mrs. Radhika, ZOO, Coimbatore, India

Mrs. Geetha, ZOO, Coimbatore India
Fundraising/Communications

Mrs. Payal B. Molur, Coimbatore, India

Subject Editors 2018-2020

Fungi

Dr. B. Shivaraju, Bengaluru, Karnataka, India

Dr. R.K. Verma, Tropical Forest Research Institute, Jabalpur, India

Dr. Vatsavaya S. Raju, Kakatiay University, Warangal, Andhra Pradesh, India

Dr. M. Krishnappa, Jnana Sahyadri, Kuvempu University, Shimoga, Karnataka, India

Dr. K.R. Sridhar, Mangalore University, Mangalagangotri, Mangalore, Karnataka, India

Dr. Gunjan Biswas, Vidyasagar University, Midnapore, West Bengal, India

\section{Plants}

Dr. G.P. Sinha, Botanical Survey of India, Allahabad, India

Dr. N.P. Balakrishnan, Ret. Joint Director, BSI, Coimbatore, India

Dr. Shonil Bhagwat, Open University and University of Oxford, UK

Prof. D.J. Bhat, Retd. Professor, Goa University, Goa, India

Dr. Ferdinando Boero, Università del Salento, Lecce, Italy

Dr. Dale R. Calder, Royal Ontaro Museum, Toronto, Ontario, Canada

Dr. Cleofas Cervancia, Univ. of Philippines Los Baños College Laguna, Philippines

Dr. F.B. Vincent Florens, University of Mauritius, Mauritius

Dr. Merlin Franco, Curtin University, Malaysia

Dr. V. Irudayaraj, St. Xavier's College, Palayamkottai, Tamil Nadu, India

Dr. B.S. Kholia, Botanical Survey of India, Gangtok, Sikkim, India

Dr. Pankaj Kumar, Kadoorie Farm and Botanic Garden Corporation, Hong Kong S.A.R., China

Dr. V. Sampath Kumar, Botanical Survey of India, Howrah, West Bengal, India

Dr. A.J. Solomon Raju, Andhra University, Visakhapatnam, India

Dr. Vijayasankar Raman, University of Mississippi, USA

Dr. B. Ravi Prasad Rao, Sri Krishnadevaraya University, Anantpur, India

Dr. K. Ravikumar, FRLHT, Bengaluru, Karnataka, India

Dr. Aparna Watve, Pune, Maharashtra, India

Dr. Qiang Liu, Xishuangbanna Tropical Botanical Garden, Yunnan, China

Dr. Noor Azhar Mohamed Shazili, Universiti Malaysia Terengganu, Kuala Terengganu, Malaysia

Dr. M.K. Vasudeva Rao, Shiv Ranjani Housing Society, Pune, Maharashtra, India

Prof. A.J. Solomon Raju, Andhra University, Visakhapatnam, India

Dr. Mandar Datar, Agharkar Research Institute, Pune, Maharashtra, India

Dr. M.K. Janarthanam, Goa University, Goa, India

Dr. K. Karthigeyan, Botanical Survey of India, India

Dr. Errol Vela, University of Montpellier, Montpellier, France

Dr. P. Lakshminarasimhan, Botanical Survey of India, Howrah, India

Dr. Larry R. Noblick, Montgomery Botanical Center, Miami, USA

Dr. K. Haridasan, Pallavur, Palakkad District, Kerala, India

Dr. Analinda Manila-Fajard, University of the Philippines Los Banos, Laguna, Philippines

Dr. P.A. Sinu, Central University of Kerala, Kasaragod, Kerala, India

Dr. Afroz Alam, Banasthali Vidyapith (accredited A grade by NAAC), Rajasthan, India

Dr. K.P. Rajesh, Zamorin's Guruvayurappan College, GA College PO, Kozhikode, Kerala, India

Dr. David E. Boufford, Harvard University Herbaria, Cambridge, MA 02138-2020, USA

Dr. Ritesh Kumar Choudhary, Agharkar Research Institute, Pune, Maharashtra, India

Dr. Navendu Page, Wildlife Institute of India, Chandrabani, Dehradun, Uttarakhand, India

\section{Invertebrates}

Dr. R.K. Avasthi, Rohtak University, Haryana, India

Dr. D.B. Bastawade, Maharashtra, India

Dr. Partha Pratim Bhattacharjee, Tripura University, Suryamaninagar, India

Dr. Kailash Chandra, Zoological Survey of India, Jabalpur, Madhya Pradesh, India

Dr. Ansie Dippenaar-Schoeman, University of Pretoria, Queenswood, South Africa

Dr. Rory Dow, National Museum of natural History Naturalis, The Netherlands

Dr. Brian Fisher, California Academy of Sciences, USA

Dr. Richard Gallon, llandudno, North Wales, LL30 1UP

Dr. Hemant V. Ghate, Modern College, Pune, India

Dr. M. Monwar Hossain, Jahangirnagar University, Dhaka, Bangladesh

Mr. Jatishwor Singh Irungbam, Biology Centre CAS, Branišovská, Czech Republic.

Dr. Ian J. Kitching, Natural History Museum, Cromwell Road, UK

Dr. George Mathew, Kerala Forest Research Institute, Peechi, India

For Focus, Scope, Aims, and Policies, visit https://threatenedtaxa.org/index.php/JoTT/aims_scope
For Article Submission Guidelines, visit https://threatenedtaxa.org/index.php/JoTT/about/submissions
For Policies against Scientific Misconduct, visit https://threatenedtaxa.org/index.php/JoTT/policies_various

continued on the back inside cover 


\title{
Macrolichens of Mathikettan Shola National Park, Western Ghats: a preliminary investigation with some new records
}

\author{
Aswathi Anilkumar ${ }^{1}$ (D), Stephen Sequeira ${ }^{2}$ (D) Arun $^{\text {Christy }}{ }^{3}$ (D) \& S.M. Arsha ${ }^{4}$ (D) \\ ${ }^{1-4}$ Lichenology lab, Post-Graduate and Research Department of Botany, Maharaja's College, Ernakulam, Kerala 682011, India. \\ ${ }^{1}$ aswathianilkumar210@gmail.com (corresponding author), ${ }^{2}$ step@rediffmail.com, ${ }^{3}$ arunchristysebastian03@gmail.com, \\ ${ }^{4}$ arsha.smohan@gmail.com
}

\begin{abstract}
An extensive survey of lichens was conducted in different parts of Mathikettan Shola National Park, and analysed 55 macrolichen species under six families. Two species were found to be new to the Indian peninsula, and five species were new to the lichen flora of
\end{abstract} Kerala.

Keywords: Biodiversity, Corticolous, Foliose, Fruiticose, lichens, Kerala, Idukki, new reports, Saxicolous.

Citation: Anilkumar, A., S. Sequeira, A. Christy \& S.M. Arsha (2022). Macrolichens of Mathikettan Shola National Park, Western Ghats: a preliminary investigation with some new records. Journal of Threatened Taxa 14(1): 20400-20405. https://doi.org/10.11609/jott.7117.14.1.20400-20405

Copyright: (c) Anilkumar et al. 2022. Creative Commons Attribution 4.0 International License. JoTT allows unrestricted use, reproduction, and distribution of this article in any medium by providing adequate credit to the author(s) and the source of publication.

Funding: UGC, New Delhi; KSCSTE, Kerala.

Competing interests: The authors declare no competing interests.

Author details: Aswathi Anilkumar is UGC- SRF at department of botany, Maharajas College, Kerala, India with research interest in taxonomy, ecology and molecular categorization of Lichens. Stephen Sequeira is currently working as Assistant Professor in Botany at Maharaja's College, Ernakulam, Kerala, India. His area of interest is taxonomy, ecology, molecular, pollution monitoring and climate change studies of lichens. Arun Christy currently working as project fellow at department of botany Maharajas College Ernakulam. He deals with taxonomic studies of lichens in Kerala and his area of interest are Lichenology, Ecology and Angiosperm taxonomy. Arsha SM is currently research scholar in department of Botany, Maharajas College. Her area of interest is molecular characterization of Parmelioid lichens.

Author contributions: AAK-conceptualization, performed field collection, formal analysis, writing original draft; SS-supervision, review, provided proper guidelines for the research; AC-review and editing draft; ASM-editing draft.

Acknowledgements: The authors are thankful to University Grants Commission (UGC), New Delhi and Kerala State Council for Science, Technology and Environment (KSCSTE), Thiruvananthapuram, Government of Kerala for the financial support for the work. The authors are grateful the Principal, Maharaja's College Ernakulam for providing the necessary facilities. Thankful to officials of Kerala Forest Department for the permission to access the area.

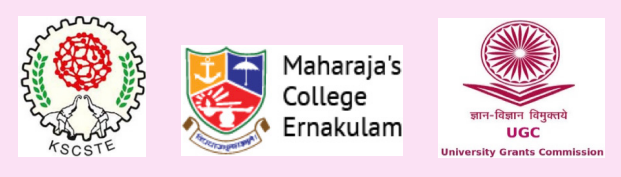




\section{INTRODUCTION}

Mathikettan Shola National Park (MSNP, 9.95010.010 $\mathrm{N}$ and 76.23-77.26 E), located in the high ranges of southern Western Ghats with an area of 1,282 ha falls under Poopara village of Ubumbanchola taluk in Idukki district, Kerala (Image 1). Altitude of the area ranges from 1,200-1,984 $\mathrm{m}$ in the highest peak-Kattamala-of the national park. The area represents a unique montane evergreen forest ecosystem with several endemic species-63 species of trees, 163 herbs and shrubs, and 15 species of climbers (Management Plan MSNP 2009).

The climatic conditions and the presence of forests intermingled with grasslands make MSNP suitable for the luxurious growth of lichens. However, to date no substantial work on lichens has reported on this unique area. Fragmentary lichen collections from different parts of Kerala (Kumar et al. 1999, 2000; Biju et al. 2010, 2012, 2014; Sonia et al. 2018, 2020) have not covered several interesting areas, including Wildlife Sanctuaries, national parks, mangrove forests, and cultivated areas (Sequiera 2003, 2005, 2008; Kumar et al. 2008). This report presents preliminary observations of macrolichens from a hitherto unrecorded area of MSNP, Idukki, Kerala.

\section{MATERIALS AND METHODS}

Data collection: An extensive survey of lichens was conducted in different parts of MSNP during the period of June 2019 to February 2020. Collection was made from Choondal (1,200-1,600 m), Karadippara (1,200 $\mathrm{m})$, and Shivanpara $(1,400 \mathrm{~m})$ area of the national park. Substrate of collection, altitude and names of trees along with the lichen population was noted from each locality. The collected specimens were numbered, air dried and herbariums were prepared as per the standard method.

Identification: Collected specimens were identified based on morphological observation and comparison with published keys and descriptions (Awasthi 2007; Mishra \& Upreti 2017). Species confirmation was done using various chemical colour tests such as potassium hydroxide $(K)$, paraphenylene diamine $(P)$, calcium hypochlorite (C), potassium iodide and thin layer chromatography (TLC) using a solvent containing toluene, dioxane, and acetic acid (TDA).

\section{RESULTS AND DISCUSSION}

More than 500 specimens were collected from the study area in MSNP. Critical analysis of the specimens revealed 55 macrolichen species under 17 genera belonging to six families; eight species were fruticose $(13 \%)$ and $47(87 \%)$ were foliose in nature. There was a maximum diversity of corticolous lichens represented by 47 species $(87 \%)$, with the rest being saxicolous in nature $(13 \%)$. Numerical representation of the taxa recorded is presented in Table 1 . Family Parmeliaceae was predominant with 25 species from seven genera, followed by Physciaceae with 11 species from two genera, Peltigeraceae with nine species from three genera, Collemataceae with four species from two genera, Coccocarpiaceae with three species from one genus, and Ramalinaceae with one species. Among 17 genera, Parmotrema and Heterodermia were found to be dominant in the study area with nine species each followed by Usnea (6 species), Sticta, Psuedocyphellaria and Hypotrachyna with four species each, Coccocarpia, Ramalina and Leptogium with two species each, Phaeophyscia, Xanthoparmelia and Canoparmelia with two species each, Lobaria, Collema, Physcia, Myelochroa, Parmelina with one species each. Among the 55 species reported from the national park, two species were new to peninsular India and five species were found to be new to the lichen flora of Kerala.

\section{New reports of lichens to Peninsular India}

1. Leptogium furfuraceum (Harm.) Sierk.

Thallus corticolous, weekly adnate, dark brown to slate gray, lobes flabellate to orbicular, $3-5 \mathrm{~cm}$ wide, margins entire to lacerate; upper surface distinctly wrinkled, isidiate; isidia globular to clavate, laminal to marginal; lower surface with white tomentose on lower surface; apothecia absent (Image 2).

Specimen examined: India, Kerala, Idukki, Mathikettan Shola National Park, $10.009 \mathrm{~N}$ to $77.239 \mathrm{E}, 1,458 \mathrm{~m}$, on bark, July, Aswathi Anilkumar (2442).

The species has an earlier record from Uttarakhand state (Awasthi 2007). The present collection shows its extended distribution in peninsular India.

\section{Parmelina usambarensis (Steiner \& Zahlbr.) Hale}

Thallus saxicolous, loosely attached on rock, whitish mineral grey, 3-5 cm across; lobes sublinear to rotund, 5-6 mm wide, divaricately branched, ciliate, sparsely to densely isidiate; isidia cylindrical, simple to branched; medulla white; lower surface shiny black, rhizines black, simple, $1 \mathrm{~mm}$ long; apothecia not present (Image 3). 


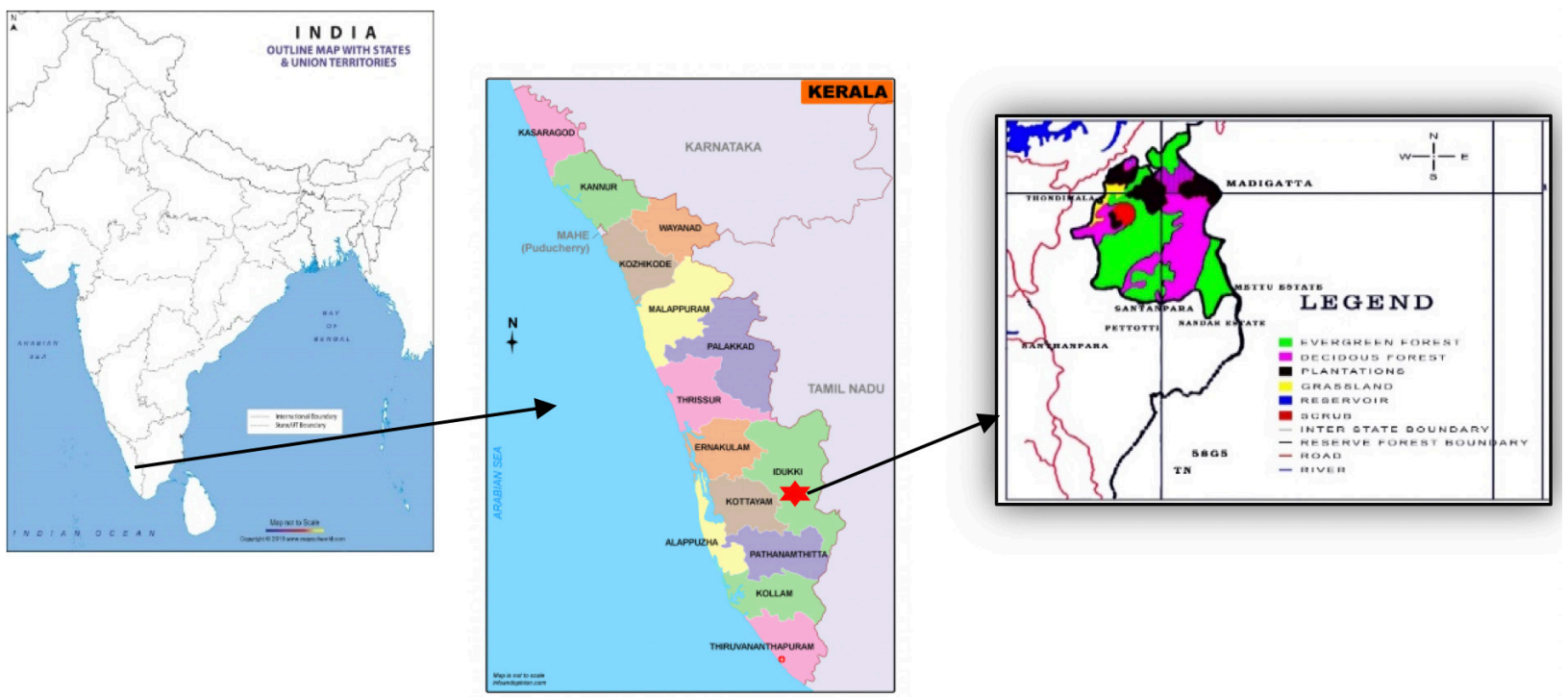

Image 1. Vegetative map of study area. Map source: www.mapsofindia.com; www.infoandopinion.com

Cortex $\mathrm{K}^{+}$yellow; medulla $\mathrm{K}^{+}$red, $\mathrm{C}^{-}, \mathrm{KC}^{-}, \mathrm{P}^{+}$red.

Specimen examined: India, Kerala, Idukki, Mathikettan Shola National Park, $10.009 \mathrm{~N}$ to $77.245 \mathrm{E}, 1,603 \mathrm{~m}$, on rock, July, Aswathi Anilkumar (2436).

This species has been reported earlier from eastern Himalaya and from Manipur state. The present collection from the study area shows its distribution in peninsular India.

\section{New reports of lichen from Kerala}

\section{Xanthoparmelia congensis (Stein) Hale}

Thallus saxicolous, very tightly adnate to the rock, foliose but centrally subcrustose, $1.5-4 \mathrm{~cm}$ across; lobes sub dichotomously branched, sublinear, 0.05-0.4mm wide; upper side greenish yellow, shiny at apices, dull at the center, aeriolate, isidiate; isidia pale, simple, globose often bursting open at top not forming soredia; medulla white; apothecia not seen, lower side black, shiny, rhizinate; apothecia not seen (Image 4).

Medulla $\mathrm{K}^{+}$yellow, $\mathrm{C}^{-}, \mathrm{KC}^{-}, \mathrm{P}^{+}$dark orange; stictic, constictic, and norstictic acid present.

Specimen examined: India, Kerala, Idukki, Mathikettan Shola National Park, $10.009 \mathrm{~N}$ to $77.242 \mathrm{E}, 1,603 \mathrm{~m}$, on rock, July, Aswathi Anilkumar (2498).

Found distributed in the state of Karnataka, Madhya Pradesh, Tamil Nadu, and Uttarakhand. The present collection confirms its extended distribution to the state of Kerala.

\section{Xanthoparmelia psuedocongensis Hale}

Thallus saxicolous, subcrustose, very tightly adnate to the substratum, 7c m across; lobes sublinear to rotund, 0.7-0.9 mm wide, black rimmed; upper surface yellowishgreen, shiny in periphery, dull in center, isidiate; isidia cylindrical, simple, black tipped; medulla white; lower surface black, shiny, rhizinate, rhizines black. Apothecia absent (Image 5).

Cortex $\mathrm{K}^{-}$; Medulla $\mathrm{K}^{+}$yellow, $\mathrm{C}^{-}, \mathrm{KC}^{-}$, $\mathrm{P}^{+}$orange; Stictic, Constictic and norstictic acid present.

Specimen examined: India, Kerala, Idukki, Mathikettan Shola National Park, $10.006 \mathrm{~N}$ to $77.243 \mathrm{E} 1,582 \mathrm{~m}$, on rock, July, Aswathi Anilkumar \& Stephen Sequeira (2497).

Recorded from Madhya Pradesh and Rajasthan.

\section{Parmotrema chinense (Osbeck) Hale \& Ahti}

Corticolous, less adnate, 3-5 cm across; lobes irregular, 1-4 $\mathrm{mm}$ wide; upper surface white grey to dark grey, margins entire, ciliate, emaculate, smooth, sorediate; Soredia marginal to submarginal; medulla white; lower surface black in centre, shiny, rhizinate, brown towards margin, erhizinate; apothecia not seen (Image 6).

Cortex $\mathrm{K}^{+}$yellow, medulla $\mathrm{K}^{+}$yellow, $\mathrm{C}^{-}, \mathrm{KC}^{-}, \mathrm{P}^{+}$pale orange; atranorin, stictic, and constictic acids present.

Specimen examined: India, Kerala, Idukki, Mathikettan Shola National Park, $10.008 \mathrm{~N}$ to $77.245 \mathrm{E}, 1,606 \mathrm{~m}$, on bark, July, Aswathi Anilkumar (2427).

Awasthi (2007) reported the occurrence of this species from Nilgiri and Palni hills of Tamil Nadu. The present collection confirms its extended distribution to the state of Kerala. 

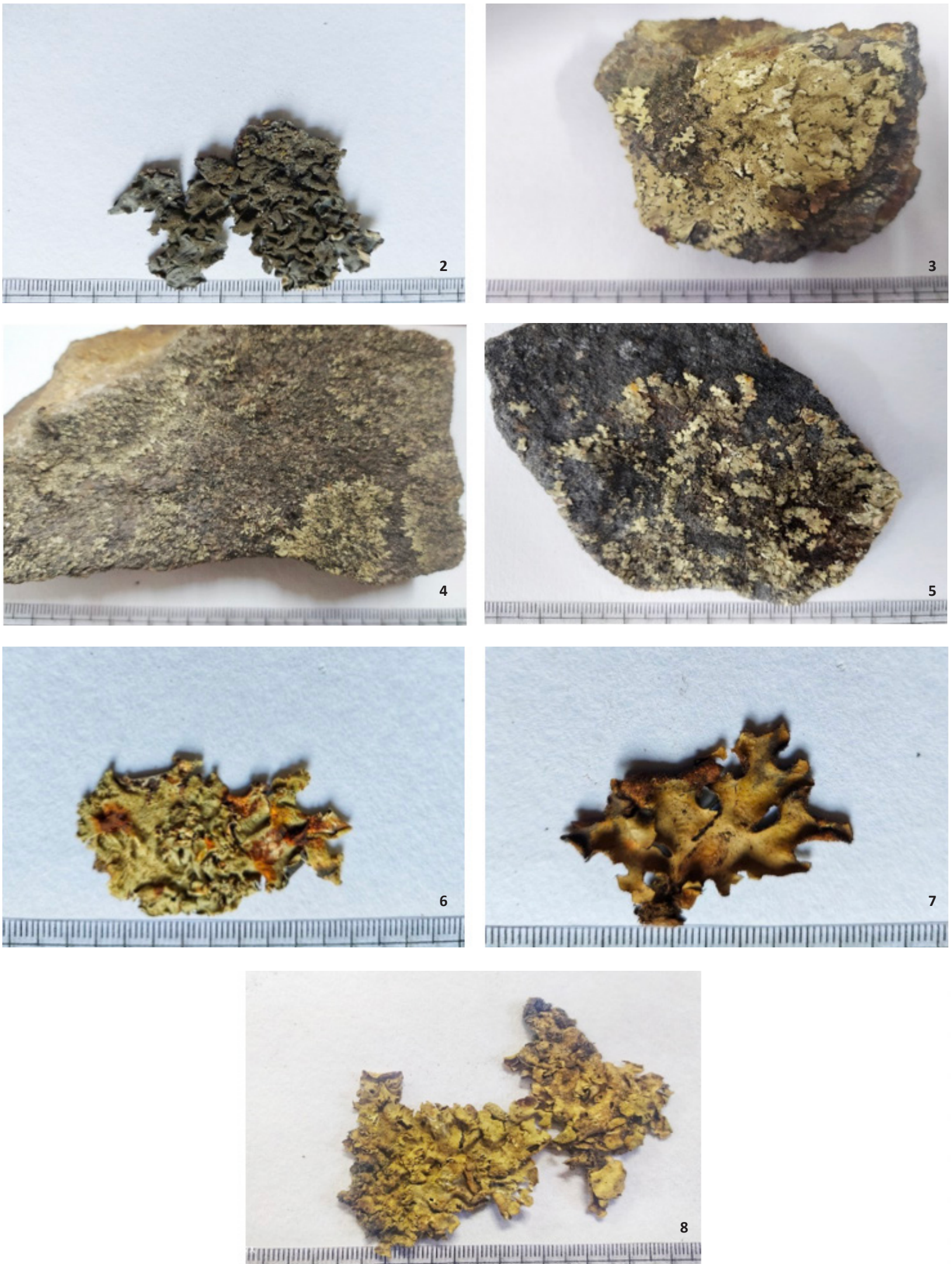

Image 2-8. New reports to lichen flora of peninsular India and Kerala: 2-Leptogium furfuraceum | 3-Parmelina usambarensis | 4Xanthoparmelia congensis | 5-Xanthoparmelia psuedocongensis | 6-Parmotrema chinense | 7-Sticta duplolimbata | 8-Lobaria japonica. (C) Aswathi Anilkumar and Stephen Sequeira. 
Table 1. Enumeration of macro lichens from Mathikettan Shola National Park.

\begin{tabular}{|c|c|c|c|}
\hline & Species & Family & $\begin{array}{l}\text { Thallus } \\
\text { type and } \\
\text { substratum }\end{array}$ \\
\hline 1 & $\begin{array}{l}\text { Coccocarpia palmicola } \\
\text { (Spreng.) Arvidss. \& D.J. } \\
\text { Galloway }\end{array}$ & Coccocarpiaceae & $\begin{array}{l}\text { Foliose } \\
\text { Saxicolous }\end{array}$ \\
\hline 2 & $\begin{array}{l}\text { Coccocarpia pellita (Ach.) } \\
\text { Mull. Arg. Em. R. Sant. }\end{array}$ & Coccocarpiaceae & $\begin{array}{l}\text { Foliose } \\
\text { Saxicolous }\end{array}$ \\
\hline 3 & Coccocarpia sp. & Coccocarpiaceae & $\begin{array}{l}\text { Foliose } \\
\text { Saxicolous }\end{array}$ \\
\hline 4 & $\begin{array}{l}\text { Collema auriforme (With.) } \\
\text { Coppins \& J.R. Laundon }\end{array}$ & Collemataceae & $\begin{array}{l}\text { Foliose } \\
\text { Corticolous }\end{array}$ \\
\hline 5 & $\begin{array}{l}\text { Leptogium cyanescens } \\
\text { (Rabenh.) Körb. }\end{array}$ & Collemataceae & $\begin{array}{l}\text { Foliose } \\
\text { Corticolous }\end{array}$ \\
\hline 6 & $\begin{array}{l}\text { Leptogium marginella (Sw.) } \\
\text { Gray }\end{array}$ & Collemataceae & $\begin{array}{l}\text { Foliose } \\
\text { Corticolous }\end{array}$ \\
\hline 7 & $\begin{array}{l}\text { Lobaria japonica (Zahlbr). } \\
\text { Asahina }\end{array}$ & Peltigeraceae & $\begin{array}{l}\text { Foliose } \\
\text { Corticolous }\end{array}$ \\
\hline 8 & $\begin{array}{l}\text { Psuedocyphellaria } \\
\text { argyraceae (Bory ex Delise) } \\
\text { Vain. }\end{array}$ & Peltigeraceae & $\begin{array}{l}\text { Foliose } \\
\text { Corticolous }\end{array}$ \\
\hline 9 & $\begin{array}{l}\text { Psuedocyphellaria aurata } \\
\text { (Sm. Ex Ach.) Vain }\end{array}$ & Peltigeraceae & $\begin{array}{l}\text { Foliose } \\
\text { Corticolous }\end{array}$ \\
\hline 10 & $\begin{array}{l}\text { Psuedocyphellaria } \\
\text { ceylonensis H. Magn. }\end{array}$ & Peltigeraceae & $\begin{array}{l}\text { Foliose } \\
\text { Corticolous }\end{array}$ \\
\hline 11 & $\begin{array}{l}\text { Psuedocyphellaria crocata } \\
\text { (L.) Vain }\end{array}$ & Peltigeraceae & $\begin{array}{l}\text { Foliose } \\
\text { Corticolous }\end{array}$ \\
\hline 12 & $\begin{array}{l}\text { Psuedocyphellaria intricata } \\
\text { (Delise) Vain }\end{array}$ & Peltigeraceae & $\begin{array}{l}\text { Foliose } \\
\text { Corticolous }\end{array}$ \\
\hline 13 & $\begin{array}{l}\text { Sticta duplolimbata (Hue) } \\
\text { Vain. }\end{array}$ & Peltigeraceae & $\begin{array}{l}\text { Foliose } \\
\text { Corticolous }\end{array}$ \\
\hline 14 & Sticta limbata (Sm.) Ach & Peltigeraceae & $\begin{array}{l}\text { Foliose } \\
\text { Corticolous }\end{array}$ \\
\hline 15 & Sticta orbicularis (R. Br.) Hue & Peltigeraceae & $\begin{array}{l}\text { Foliose } \\
\text { Corticolous }\end{array}$ \\
\hline 16 & Sticta weigelii (Ach.) Vain. & Peltigeraceae & $\begin{array}{l}\text { Foliose } \\
\text { Corticolous }\end{array}$ \\
\hline 17 & $\begin{array}{l}\text { Canoparmelia pustulescence } \\
\text { (Kurok.) Elix }\end{array}$ & Parmeliaceae & $\begin{array}{l}\text { Foliose } \\
\text { Corticolous }\end{array}$ \\
\hline 18 & $\begin{array}{l}\text { Canoparmelia texana (Tuck.) } \\
\text { Elix \& Hale }\end{array}$ & Parmeliaceae & $\begin{array}{l}\text { Foliose } \\
\text { Corticolous }\end{array}$ \\
\hline 19 & $\begin{array}{l}\text { Hypotrachyna cirrhata (Fr.) } \\
\text { Divakar, A. Crespo, Sipman, } \\
\text { Elix \& Lumbsch }\end{array}$ & Parmeliaceae & $\begin{array}{l}\text { Foliose } \\
\text { Corticolous }\end{array}$ \\
\hline 20 & $\begin{array}{l}\text { Hypotrachyna dactylifera } \\
\text { (Vain.) Hale }\end{array}$ & Parmeliaceae & $\begin{array}{l}\text { Foliose } \\
\text { Corticolous }\end{array}$ \\
\hline 21 & $\begin{array}{l}\text { Hypotrachyna infirma } \\
\text { (Kurok.) Hale }\end{array}$ & Parmeliaceae & $\begin{array}{l}\text { Foliose } \\
\text { Corticolous }\end{array}$ \\
\hline 22 & $\begin{array}{l}\text { Hypotrachyna nepalense } \\
\text { (Taylor) Divakar, A. Crespo, } \\
\text { Sipman, Elix \& Lumbsch }\end{array}$ & Parmeliaceae & $\begin{array}{l}\text { Foliose } \\
\text { Corticolous }\end{array}$ \\
\hline 23 & $\begin{array}{l}\text { Myelochroa xantholepis } \\
\text { (Mont. \& Bosch) Elix \& Hale }\end{array}$ & Parmeliaceae & $\begin{array}{l}\text { Foliose } \\
\text { Corticolous }\end{array}$ \\
\hline 24 & $\begin{array}{l}\text { Parmelina usambarensis } \\
\text { (Steiner \& Zahlbr.) Hale }\end{array}$ & Parmeliaceae & $\begin{array}{l}\text { Foliose } \\
\text { Saxicolous }\end{array}$ \\
\hline 25 & $\begin{array}{l}\text { Parmotrema chinense } \\
\text { (Osbeck) Hale \& Ahti }\end{array}$ & Parmeliaceae & $\begin{array}{l}\text { Foliose } \\
\text { Corticolous }\end{array}$ \\
\hline 26 & Parmotrema indicum Hale & Parmeliaceae & $\begin{array}{l}\text { Foliose } \\
\text { Corticolous }\end{array}$ \\
\hline
\end{tabular}

\begin{tabular}{|c|c|c|c|}
\hline 27 & $\begin{array}{l}\text { Parmotrema tinctorum } \\
\text { (Despr. ex Nyl) Hale }\end{array}$ & Parmeliaceae & $\begin{array}{l}\text { Foliose } \\
\text { Corticolous }\end{array}$ \\
\hline 28 & $\begin{array}{l}\text { Parmotrema reticulatum } \\
\text { (Taylor) Choisy }\end{array}$ & Parmeliaceae & $\begin{array}{l}\text { Foliose } \\
\text { Corticolous }\end{array}$ \\
\hline 29 & $\begin{array}{l}\text { Parmotrema crinitum (Ach.) } \\
\text { Choisy }\end{array}$ & Parmeliaceae & $\begin{array}{l}\text { Foliose } \\
\text { Corticolous }\end{array}$ \\
\hline 30 & $\begin{array}{l}\text { Parmotrema praesorediosum } \\
\text { (Nyl.) Hale }\end{array}$ & Parmeliaceae & $\begin{array}{l}\text { Foliose } \\
\text { Corticolous }\end{array}$ \\
\hline 31 & $\begin{array}{l}\text { Parmotrema hababianum } \\
\text { (Gyeln.) Hale }\end{array}$ & Parmeliaceae & $\begin{array}{l}\text { Foliose } \\
\text { Corticolous }\end{array}$ \\
\hline 32 & $\begin{array}{l}\text { Parmotrema cristiferum } \\
\text { (Taylor) Hale }\end{array}$ & Parmeliaceae & $\begin{array}{l}\text { Foliose } \\
\text { Corticolous }\end{array}$ \\
\hline 33 & $\begin{array}{l}\text { Parmotrema stuppeum } \\
\text { (Taylor) Hale }\end{array}$ & Parmeliaceae & $\begin{array}{l}\text { Foliose } \\
\text { Corticolous }\end{array}$ \\
\hline 34 & Usnea baileyi (Stirt.) Zahlbr. & Parmeliaceae & $\begin{array}{l}\text { Fruticose } \\
\text { Corticolous }\end{array}$ \\
\hline 35 & $\begin{array}{l}\text { Usnea rigidula (Stirt.) G. } \\
\text { Awasthi }\end{array}$ & Parmeliaceae & $\begin{array}{l}\text { Fruticose } \\
\text { Corticolous }\end{array}$ \\
\hline 36 & Usnea thomsonii Stirt. & Parmeliaceae & $\begin{array}{l}\text { Fruticose } \\
\text { Corticolous }\end{array}$ \\
\hline 37 & Usnea pectinate Taylor & Parmeliaceae & $\begin{array}{l}\text { Fruticose } \\
\text { Corticolous }\end{array}$ \\
\hline 38 & Usnea picta (J. Steiner) Mot. & Parmeliaceae & $\begin{array}{l}\text { Fruticose } \\
\text { Corticolous }\end{array}$ \\
\hline 39 & $\begin{array}{l}\text { Usnea subflorida (Zahlbr.) } \\
\text { Mot. }\end{array}$ & Parmeliaceae & $\begin{array}{l}\text { Fruticose } \\
\text { Corticolous }\end{array}$ \\
\hline 40 & $\begin{array}{l}\text { Xanthoparmelia congensis (B. } \\
\text { Stein) Hale }\end{array}$ & Parmeliaceae & $\begin{array}{l}\text { Foliose } \\
\text { Saxicolous }\end{array}$ \\
\hline 41 & $\begin{array}{l}\text { Xanthoparmelia } \\
\text { psuedocongensis Hale }\end{array}$ & Parmeliaceae & $\begin{array}{l}\text { Foliose } \\
\text { Saxicolous }\end{array}$ \\
\hline 42 & $\begin{array}{l}\text { Heterodermia boryi (Fée) Kr.P. } \\
\text { Singh \& S.R. Singh }\end{array}$ & Physciaceae & $\begin{array}{l}\text { Foliose } \\
\text { Corticolous }\end{array}$ \\
\hline 43 & $\begin{array}{l}\text { Heterodermia comosa } \\
\text { (Eschw.) Follman \& Redon }\end{array}$ & Physciaceae & $\begin{array}{l}\text { Foliose } \\
\text { Corticolous }\end{array}$ \\
\hline 44 & $\begin{array}{l}\text { Heterodermia hypocaesia } \\
\text { (Yasuda) D.D. Awasthi }\end{array}$ & Physciaceae & $\begin{array}{l}\text { Foliose } \\
\text { Corticolous }\end{array}$ \\
\hline 45 & $\begin{array}{l}\text { Heterodermia incana } \\
\text { (Stirton) D. D. Awasthi }\end{array}$ & Physciaceae & $\begin{array}{l}\text { Foliose } \\
\text { Corticolous }\end{array}$ \\
\hline 46 & $\begin{array}{l}\text { Heterodermia isidiophora } \\
\text { (Vain.) D.D. Awasthi }\end{array}$ & Physciaceae & $\begin{array}{l}\text { Foliose } \\
\text { Corticolous }\end{array}$ \\
\hline 47 & $\begin{array}{l}\text { Heterodermia japonica (Sato) } \\
\text { Swinsc. \& Krog }\end{array}$ & Physciaceae & $\begin{array}{l}\text { Foliose } \\
\text { Corticolous }\end{array}$ \\
\hline 48 & $\begin{array}{l}\text { Heterodermia obscurata } \\
\text { (Nyl.) Trevis. }\end{array}$ & Physciaceae & $\begin{array}{l}\text { Foliose } \\
\text { Corticolous }\end{array}$ \\
\hline 49 & $\begin{array}{l}\text { Heterodermia speciosa } \\
\text { (Wulf.) Trevis. }\end{array}$ & Physciaceae & $\begin{array}{l}\text { Foliose } \\
\text { Corticolous }\end{array}$ \\
\hline 50 & $\begin{array}{l}\text { Heterodermia togashii } \\
\text { (Kurok.) D.D. Awasthi }\end{array}$ & Physciaceae & $\begin{array}{l}\text { Foliose } \\
\text { Corticolous }\end{array}$ \\
\hline 51 & $\begin{array}{l}\text { Pheophyscia hispidula (Ach.) } \\
\text { Moberg }\end{array}$ & Physciaceae & $\begin{array}{l}\text { Foliose } \\
\text { Corticolous }\end{array}$ \\
\hline 52 & $\begin{array}{l}\text { Pheophyscia orbicularis } \\
\text { (Neck.) Moberg }\end{array}$ & Physciaceae & $\begin{array}{l}\text { Foliose } \\
\text { Corticolous }\end{array}$ \\
\hline 53 & Physcia tribacoides Nyl. & Physciaceae & $\begin{array}{l}\text { Foliose } \\
\text { Saxicolous }\end{array}$ \\
\hline 54 & Ramalina conduplicans Vain. & Ramalinaceae & $\begin{array}{l}\text { Fruticose } \\
\text { Corticolous }\end{array}$ \\
\hline 55 & Ramalina pacifica Asahina & Ramalinaceae & $\begin{array}{l}\text { Fruticose } \\
\text { Corticolous }\end{array}$ \\
\hline
\end{tabular}




\section{Sticta duplolimbata (Hue) Vain.}

Corticolous thallus, photobiont cyanobacterium, holdfast seen, foliose, $4-5 \mathrm{~cm}$ wide; upper surface yellowish-brown, glossy, ciliate, cilia black, isidiate; Isidia black, marginal; medulla off white; lower surface brown, tomentose, cyphellae yellow; apothecia not known (Image 7).

Specimen examined: India, Kerala, Idukki, Mathikettan Shola National Park, $10.007 \mathrm{~N}$ to $77.246 \mathrm{E}, 1,591 \mathrm{~m}$, on rock, July, Aswathi Anilkumar (2480).

Recently collected from Nilgris hills of Tamil Nadu (Pandit \& Sharma 2012). The present collection confirms its extended distribution to the state of Kerala.

\section{Lobaria japonica (Zahlbr). Asahina}

Thallus corticolous, loosely adnate, 5-9 cm across, yellow brown, dull, photobiont green algae; Upper surface smooth without recticulate ridges, minor wrinkles; no isidia and soredia; lower surface pale brown, tomentose, rhizinate, rhizines black; apothecia immature (Image 8).

Cortex $\mathrm{K}^{-}$; medulla $\mathrm{K}^{-}, \mathrm{C}^{-}, \mathrm{KC}^{-}$, $\mathrm{P}^{-}$. No lichen materials

Specimen examined: India, Kerala, Idukki, Mathikettan Shola National Park, $10.006 \mathrm{~N}$ to $77.243 \mathrm{E}, 1,582 \mathrm{~m}$, on rock, July, Aswathi Anilkumar (2380).

Collected from Nilgris hills of Tamil Nadu and Nagaland.

\section{CONCLUSION}

It is estimated that India supports about 2,532 lichen species under 324 genera and 78 families, including 541 endemic species (Singh \& Sinha 2010). Only about 691 species are so far reported from Kerala since only fragmentary studies have been done on lichen taxonomy from the state. This study mainly focused on survey of macro lichen species from Mathikettan Shola National Park, and the results revealed that further extensive exploratory studies may end up with new additions to lichen biota of the state, and also to the country.

\section{REFERENCES}

Awasthi, D.D. (2007). A Compendium of the Macrolichens from India Nepal and Sri Lanka. Bishen Singh Mahendra Pal Singh, Dehra Dun, India, $580 \mathrm{pp}$.

Biju, H., R.G. Bagool \& S. Nayaka (2010). Additions to the lichen flora of Kerala state 2; Parmeloid macro lichens. Jawaharlal Nehru Tropical Botanic Garden and Research Institute, Kerala, India.

Biju, H., R.G. Bagool \& S. Nayaka (2012). Additions to the lichen flora of Kerala state 2; Graphidaceae. Jawaharlal Nehru Tropical Botanic Garden and Research Institute, Kerala, India.

Biju, H., R.G. Bagool \& S. Nayaka (2014). New records of Graphidaceous lichens form Western Ghats, India. Indian Journal of Forestry 37(4): 477-481.

Management Plan Mathikettan Shola National Park (2009). First Management Plan, Mathikettan Shola National Park 2009-2018. Department of Forests and Wildlife, Govt. of Kerala.

Kumar, M. (2000). Lichen (Macrolichen) flora of Kerala part of Western Ghats. KFRI Research Report No. 194. Kerala Forest Research Institute, Peechi, Thrissur, Kerala, 212 pp.

Kumar, M. \& S. Sequiera (1999). Lichens of Western Ghats: An overview, pp. 297-331. In: Mukerji, K.G., B.P. Chamola, D.K. Upreti \& R.K. Upadhyay (eds.). Biology of Lichens. Aravali Books International, New Delhi, 419 pp.

Kumar, M. \& S. Sequiera (2008). Preliminary survey on the lower groups of plants of Neyyar Wildlife Sanctuary, Thiruvananthapuram, Kerala (India). Indian Journal of Forestry 31(2): 261-268.

Mishra, G. \& D. Upreti (2017). The lichen genus Parmotrema A. Massal. (Lecanorales, Ascomycota) from India with addition distributional records. Cryptogam Biodiversity and Assessment 2(02): 18-40.

Pandit, G. \& B. Sharma (2012). New records in the lichen family Lobariaceae from the Western Ghats of India. Mycosphere 3(4): 430-435. https://doi.org/10.5943/mycosphere/3/4/6

Sequiera, S. (2003). Taxonomy and Ecology of lichens of Silent Valley National Park, southern Western Ghats, India. PhD. Thesis, FRI, Dehra Dun.

Sequiera, S., M. Remesh \& M. Kumar (2005). Lichens: the unsung members of Kerala forests, pp. 181-182. In: Muthunayagam, A.E. (ed.). Proceedings of $17^{\text {th }}$ Kerala Science Congress 2005. Kerala State Council for Science, Technology \& Environment.

Sequiera, S. \& M. Kumar (2008). Epiphyte host relationship of Macrolichens in the tropical wet evergreen forest of Silent Valley National Park, Western Ghats of India. Tropical Ecology 49(2): 211224.

Singh, K.P. \& G.P. Sinha (2010). Indian Lichens: An Annotated Checklist. Botanical Survey of India, Kolkata, 571 pp.

Zachariah, S.A., S. Nayaka, S. Joseph, P. Gupta, S. Thomas \& S.K. Varghese (2018). New and noteworthy records of lichens from Pathanamthitta district, Kerala, India. Studies in Fungi 3(1): 349-356.

Zachariah, S.A., S. Nayaka, S. Joseph, P. Gupta, S. Thomas \& S.K. Varghese (2018). Eleven new records of lichens to the state of Kerala, India. Journal of Threatened Taxa 12(10): 16402-16406. https://doi. org/10.11609/jott.5475.12.10.16402-16406 
Dr. John Noyes, Natural History Museum, London, UK

Dr. Albert G. Orr, Griffith University, Nathan, Australia

Dr. Sameer Padhye, Katholieke Universiteit Leuven, Belgium

Dr. Nancy van der Poorten, Toronto, Canada

Dr. Kareen Schnabel, NIWA, Wellington, New Zealand

Dr. R.M. Sharma, (Retd.) Scientist, Zoological Survey of India, Pune, India

Dr. Manju Siliwal, WILD, Coimbatore, Tamil Nadu, India

Dr. G.P. Sinha, Botanical Survey of India, Allahabad, India

Dr. K.A. Subramanian, Zoological Survey of India, New Alipore, Kolkata, India

Dr. P.M. Sureshan, Zoological Survey of India, Kozhikode, Kerala, India

Dr. R. Varatharajan, Manipur University, Imphal, Manipur, India

Dr. Eduard Vives, Museu de Ciències Naturals de Barcelona, Terrassa, Spain

Dr. James Young, Hong Kong Lepidopterists' Society, Hong Kong

Dr. R. Sundararaj, Institute of Wood Science \& Technology, Bengaluru, India

Dr. M. Nithyanandan, Environmental Department, La Ala Al Kuwait Real Estate. Co. K.S.C.,

Kuwait

Dr. Himender Bharti, Punjabi University, Punjab, India

Mr. Purnendu Roy, London, UK

Dr. Saito Motoki, The Butterfly Society of Japan, Tokyo, Japan

Dr. Sanjay Sondhi, TITLI TRUST, Kalpavriksh, Dehradun, India

Dr. Nguyen Thi Phuong Lien, Vietnam Academy of Science and Technology, Hanoi, Vietnam

Dr. Nitin Kulkarni, Tropical Research Institute, Jabalpur, India

Dr. Robin Wen Jiang Ngiam, National Parks Board, Singapore

Dr. Lional Monod, Natural History Museum of Geneva, Genève, Switzerland.

Dr. Asheesh Shivam, Nehru Gram Bharti University, Allahabad, India

Dr. Rosana Moreira da Rocha, Universidade Federal do Paraná, Curitiba, Brasi

Dr. Kurt R. Arnold, North Dakota State University, Saxony, Germany

Dr. James M. Carpenter, American Museum of Natural History, New York, USA

Dr. David M. Claborn, Missouri State University, Springfield, USA

Dr. Kareen Schnabel, Marine Biologist, Wellington, New Zealand

Dr. Amazonas Chagas Júnior, Universidade Federal de Mato Grosso, Cuiabá, Brasil

Mr. Monsoon Jyoti Gogoi, Assam University, Silchar, Assam, India

Dr. Heo Chong Chin, Universiti Teknologi MARA (UiTM), Selangor, Malaysia

Dr. R.J. Shiel, University of Adelaide, SA 5005, Australia

Dr. Siddharth Kulkarni, The George Washington University, Washington, USA

Dr. Priyadarsanan Dharma Rajan, ATREE, Bengaluru, India

Dr. Phil Alderslade, CSIRO Marine And Atmospheric Research, Hobart, Australia

Dr. John E.N. Veron, Coral Reef Research, Townsville, Australia

Dr. Daniel Whitmore, State Museum of Natural History Stuttgart, Rosenstein, Germany.

Dr. Yu-Feng Hsu, National Taiwan Normal University, Taipei City, Taiwan

Dr. Keith V. Wolfe, Antioch, California, USA

Dr. Siddharth Kulkarni, The Hormiga Lab, The George Washington University, Washington,

D.C., USA

Dr. Tomas Ditrich, Faculty of Education, University of South Bohemia in Ceske

Budejovice, Czech Republic

Dr. Mihaly Foldvari, Natural History Museum, University of Oslo, Norway

Dr. V.P. Uniyal, Wildlife Institute of India, Dehradun, Uttarakhand 248001, India

Dr. John T.D. Caleb, Zoological Survey of India, Kolkata, West Bengal, India

Dr. Priyadarsanan Dharma Rajan, Ashoka Trust for Research in Ecology and the Environment

(ATREE), Royal Enclave, Bangalore, Karnataka, India

\section{Fishes}

Dr. Neelesh Dahanukar, IISER, Pune, Maharashtra, India

Dr. Topiltzin Contreras MacBeath, Universidad Autónoma del estado de Morelos, México

Dr. Heok Hee Ng, National University of Singapore, Science Drive, Singapore

Dr. Rajeev Raghavan, St. Albert's College, Kochi, Kerala, India

Dr. Robert D. Sluka, Chiltern Gateway Project, A Rocha UK, Southall, Middlesex, UK

Dr. E. Vivekanandan, Central Marine Fisheries Research Institute, Chennai, India

Dr. Davor Zanella, University of Zagreb, Zagreb, Croatia

Dr. A. Biju Kumar, University of Kerala, Thiruvananthapuram, Kerala, India

Dr. Akhilesh K.V., ICAR-Central Marine Fisheries Research Institute, Mumbai Research

Centre, Mumbai, Maharashtra, India

Dr. J.A. Johnson, Wildlife Institute of India, Dehradun, Uttarakhand, India

Amphibians

Dr. Sushil K. Dutta, Indian Institute of Science, Bengaluru, Karnataka, India

Dr. Annemarie Ohler, Muséum national d'Histoire naturelle, Paris, France

\section{Reptiles}

Dr. Gernot Vogel, Heidelberg, Germany

Dr. Raju Vyas, Vadodara, Gujarat, India

Dr. Pritpal S. Soorae, Environment Agency, Abu Dubai, UAE.

Prof. Dr. Wayne J. Fuller, Near East University, Mersin, Turkey

Prof. Chandrashekher U. Rivonker, Goa University, Taleigao Plateau, Goa. India

Dr. S.R. Ganesh, Chennai Snake Park, Chennai, Tamil Nadu, India

Dr. Himansu Sekhar Das, Terrestrial \& Marine Biodiversity, Abu Dhabi, UAE
Birds

Dr. Hem Sagar Baral, Charles Sturt University, NSW Australia

Dr. Chris Bowden, Royal Society for the Protection of Birds, Sandy, UK

Dr. Priya Davidar, Pondicherry University, Kalapet, Puducherry, India

Dr. J.W. Duckworth, IUCN SSC, Bath, UK

Dr. Rajah Jayapal, SACON, Coimbatore, Tamil Nadu, India

Dr. Rajiv S. Kalsi, M.L.N. College, Yamuna Nagar, Haryana, India

Dr. V. Santharam, Rishi Valley Education Centre, Chittoor Dt., Andhra Pradesh, India

Dr. S. Balachandran, Bombay Natural History Society, Mumbai, India

Mr. J. Praveen, Bengaluru, India

Dr. C. Srinivasulu, Osmania University, Hyderabad, India

Dr. K.S. Gopi Sundar, International Crane Foundation, Baraboo, USA

Dr. Gombobaatar Sundev, Professor of Ornithology, Ulaanbaatar, Mongolia

Prof. Reuven Yosef, International Birding \& Research Centre, Eilat, Israel

Dr. Taej Mundkur, Wetlands International, Wageningen, The Netherlands

Dr. Carol Inskipp, Bishop Auckland Co., Durham, UK

Dr. Tim Inskipp, Bishop Auckland Co, Durham, UK

Dr. V. Gokula, National College, Tiruchirappalli, Tamil Nadu, India

Dr. Arkady Lelej, Russian Academy of Sciences, Vladivostok, Russia

Dr. Simon Dowell, Science Director, Chester Zoo, UK

Dr. Mário Gabriel Santiago dos Santos, Universidade de Trás-os-Montes e Alto Douro,

Quinta de Prados, Vila Real, Portugal

Dr. Grant Connette, Smithsonian Institution, Royal, VA, USA

Dr. M. Zafar-ul Islam, Prince Saud Al Faisal Wildlife Research Center, Taif, Saudi Arabia

Mammals

Dr. Giovanni Amori, CNR - Institute of Ecosystem Studies, Rome, Italy

Dr. Anwaruddin Chowdhury, Guwahati, India

Dr. David Mallon, Zoological Society of London, UK

Dr. Shomita Mukherjee, SACON, Coimbatore, Tamil Nadu, India

Dr. Angie Appel, Wild Cat Network, Germany

Dr. P.O. Nameer, Kerala Agricultural University, Thrissur, Kerala, India

Dr. Ian Redmond, UNEP Convention on Migratory Species, Lansdown, UK

Dr. Heidi S. Riddle, Riddle's Elephant and Wildlife Sanctuary, Arkansas, USA

Dr. Karin Schwartz, George Mason University, Fairfax, Virginia.

Dr. Lala A.K. Singh, Bhubaneswar, Orissa, India

Dr. Mewa Singh, Mysore University, Mysore, India

Dr. Paul Racey, University of Exeter, Devon, UK

Dr. Honnavalli N. Kumara, SACON, Anaikatty P.O., Coimbatore, Tamil Nadu, India

Dr. Nishith Dharaiya, HNG University, Patan, Gujarat, India

Dr. Spartaco Gippoliti, Socio Onorario Società Italiana per la Storia della Fauna "Giuseppe

Altobello", Rome, Italy

Dr. Justus Joshua, Green Future Foundation, Tiruchirapalli, Tamil Nadu, India

Dr. H. Raghuram, The American College, Madurai, Tamil Nadu, India

Dr. Paul Bates, Harison Institute, Kent, UK

Dr. Jim Sanderson, Small Wild Cat Conservation Foundation, Hartford, USA

Dr. Dan Challender, University of Kent, Canterbury, UK

Dr. David Mallon, Manchester Metropolitan University, Derbyshire, UK

Dr. Brian L. Cypher, California State University-Stanislaus, Bakersfield, CA

Dr. S.S. Talmale, Zoological Survey of India, Pune, Maharashtra, India

Prof. Karan Bahadur Shah, Budhanilakantha Municipality, Kathmandu, Nepal

Dr. Susan Cheyne, Borneo Nature Foundation International, Palangkaraja, Indonesia

Dr. Hemanta Kafley, Wildlife Sciences, Tarleton State University, Texas, USA

\section{Other Disciplines}

Dr. Aniruddha Belsare, Columbia MO 65203, USA (Veterinary)

Dr. Mandar S. Paingankar, University of Pune, Pune, Maharashtra, India (Molecular)

Dr. Jack Tordoff, Critical Ecosystem Partnership Fund, Arlington, USA (Communities)

Dr. Ulrike Streicher, University of Oregon, Eugene, USA (Veterinary)

Dr. Hari Balasubramanian, EcoAdvisors, Nova Scotia, Canada (Communities)

Dr. Rayanna Hellem Santos Bezerra, Universidade Federal de Sergipe, São Cristóvão, Brazil

Dr. Jamie R. Wood, Landcare Research, Canterbury, New Zealand

Dr. Wendy Collinson-Jonker, Endangered Wildlife Trust, Gauteng, South Africa

Dr. Rajeshkumar G. Jani, Anand Agricultural University, Anand, Gujarat, India

Dr. O.N. Tiwari, Senior Scientist, ICAR-Indian Agricultural Research Institute (IARI), New

Delhi, India

Dr. L.D. Singla, Guru Angad Dev Veterinary and Animal Sciences University, Ludhiana, India

Dr. Rupika S. Rajakaruna, University of Peradeniya, Peradeniya, Sri Lanka

Dr. Bahar Baviskar, Wild-CER, Nagpur, Maharashtra 440013, India

Reviewers 2018-2020

Due to pausity of space, the list of reviewers for $2018-2020$ is available online.

The opinions expressed by the authors do not reflect the views of the Journal of Threatened Taxa, Wildlife Information Liaison Development Society, Zoo Outreach Organization, or any of the partners. The journal, the publisher, the host, and the partners are not responsible for the accuracy of the political boundaries shown in the maps by the authors.

Journal of Threatened Taxa is indexed/abstracted in Bibliography of Systematic Mycology, Biological Abstracts, BIOSIS Previews, CAB Abstracts, EBSCO, Google Scholar, Index Copernicus, Index Fungorum, JournalSeek, National Academy of Agricultural Sciences, NewJour, OCLC WorldCat, SCOPUS, Stanford University Libraries, Virtual Library of Biology, Zoological Records.

NAAS rating (India) 5.64
Print copies of the Journal are available at cost. Write to:

The Managing Editor, JoTT,

c/o Wildlife Information Liaison Development Society,

No. 12, Thiruvannamalai Nagar, Saravanampatti - Kalapatti Road,

Saravanampatti, Coimbatore, Tamil Nadu 641035, India

ravi@threatenedtaxa.org 


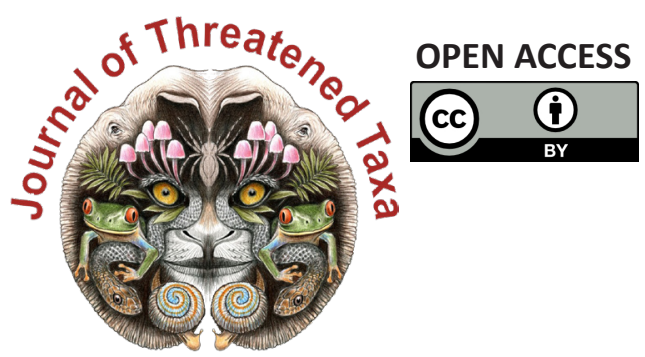

www.threatenedtaxa.org

The Journal of Threatened Taxa (JoTT) is dedicated to building evidence for conservation globally by publishing peer-reviewed articles online every month at a reasonably rapid rate at www.threatenedtaxa.org. All articles published in JoTT are registered under Creative Commons Attribution 4.0 International License unless otherwise mentioned. JoTT allows allows unrestricted use, reproduction, and distribution of articles in any medium by providing adequate credit to the author(s) and the source of publication.

\section{ISSN $0974-7907$ (Online) | ISSN $0974-7893$ (Print)}

\section{January 2022 | Vol. 14 | No. 1 | Pages: 20311-20538 \\ Date of Publication: 26 January 2022 (Online \& Print) DOI: 10.11609/jott.2022.14.1.20311-20538}

Articles

Estimating the completeness of orchid checklists and atlases: a case study from southern Italy

- Antonio Croce, Pp. 20311-20322

A floristic survey across three coniferous forests of Kashmir Himalaya, India - a checklist

- Ashaq Ahmad Dar, Akhtar Hussain Malik \& Narayanaswamy Parthasarathy, Pp. 20323-20345

Associations of butterflies across different forest types in Uttarakhand, western Himalaya, India: implications for conservation planning

- Arun Pratap Singh, Pp. 20346-20370

Comparison of bird diversity in protected and non-protected wetlands of western lowland of Nepal

- Jagan Nath Adhikari, Janak Raj Khatiwada, Dipendra Adhikari, Suman Sapkota, Bishnu Prasad Bhattarai, Deepak Rijal \& Lila Nath Sharma, Pp. 20371-20386

Local hunting practices and perceptions regarding the distribution and ecological role of the Large Flying Fox (Chiroptera: Pteropodidae: Pteropus vampyrus) in western Sarawak, Malaysian Borneo

- Jayasilan Mohd-Azlan, Joon Yee Yong, Nabila Norshuhadah Mohd Hazzrol, Philovenny Pengiran, Arianti Atong \& Sheema Abdul Aziz, Pp. 20387-20399

\section{Communications}

Macrolichens of Mathikettan Shola National Park, Western Ghats: a preliminary investigation with some new records

- Aswathi Anilkumar, Stephen Sequeira, Arun Christy \& S.M. Arsha, Pp. 20400-20405

New distribution record of globally threatened Ocean Turf Grass Halophila beccarii Ascherson, 1871 from the North Andaman Islands highlights the importance of seagrass exploratory surveys

- Swapnali Gole, Prasad Gaidhani, Srabani Bose, Anant Pande, Jeyaraj Antony Johnson \& Kuppusamy Sivakumar, Pp. 20406-20412

An inventory of new orchid (Orchidaceae) records from Kozhikode, Kerala, India - M. Sulaiman, C. Murugan \& M.U. Sharief, Pp. 20413-20425

Abundance and spatial distribution analyses of Stemonoporus moonii Thwaites (Dipterocarpaceae) - a critically endangered species endemic to Sri Lanka - K.A.M.R.P. Atapattu, H.D.D.C.K. Perera, H.S. Kathriarachchi \& A.R. Gunawardena, Pp. 20426-20432

Plant diversity of Point Calimere Wildlife Sanctuary and fodder species grazed by the Blackbuck Antilope cervicapra L.

- Ashutosh Kumar Upadhyay, A. Andrew Emmanuel, Ansa Sarah Varghese \&

D. Narasimhan, Pp. 20433-20443

Raptors observed (1983-2016) in National Chambal Gharial Sanctuary: semi-arid biogeographic region suggestions for parametric studies on ecological continuity in Khathiar-Gir Ecoregion, India

- L.A.K. Singh, R.K. Sharma \& Udayan Rao Pawar, Pp. 20444-20460

Nesting success of Sharpe's Longclaw (Macronyx sharpei Jackson, 1904) around the grasslands of lake Ol'bolossat Nyandarua, Kenya

- Hamisi Ann Risper, Charles M. Warui \& Peter Njoroge, Pp. 20461-20468

Population, distribution and diet composition of Smooth-coated Otter Lutrogale perspicillata Geoffroy, 1826 in Hosur and Dharmapuri Forest Divisions, India - Nagarajan Baskaran, Raman Sivaraj Sundarraj \& Raveendranathanpillai Sanil, Pp. 20469-20477

Utilization of home garden crops by primates and current status of human-primate interface at Galigamuwa Divisional Secretariat Division in Kegalle District, Sri Lanka

- Charmalie Anuradhie Dona Nahallage, Dahanakge Ayesha Madushani Dasanayake, Dilan Thisaru Hewamanna \& Dissanayakalage Tharaka Harshani Ananda, Pp. 2047820487
Revival of Eastern Swamp Deer Rucervus duvaucelii ranjitsinhi (Groves, 1982) in Manas National Park of Assam, India

- Nazrul Islam, Aftab Ahmed, Rathin Barman, Sanatan Deka, Bhaskar Choudhury, Prasanta Kumar Saikia \& Jyotishman Deka, Pp. 20488-20493

Trypanosoma evansi infection in a captive Indian Wolf Canis lupus pallipes - molecular diagnosis and therapy

- Manojita Dash, Sarat Kumar Sahu, Santosh Kumar Gupta, Niranjana Sahoo \& Debarat Mohapatra, Pp. 20494-20499

View Point

COVID-19 and civil unrest undoing steady gains in karst conservation and herpetological research in Myanmar, and an impediment to progress - Evan S.H. Quah, Lee L. Grismer, Perry L. Wood, Jr., Aung Lin \& Myint Kyaw Thura, Pp. 20500-20502

\section{Short Communications}

Morphological characterization and mt DNA barcode of a tiger moth species, Asota ficus (Fabricius, 1775) (Lepidoptera: Noctuoidea: Erebidae: Aganainae) from India - Aparna Sureshchandra Kalawate, K.P. Dinesh \& A. Shabnam, Pp. 20503-20510

Distribution of Smooth-coated Otters Lutrogale perspicillata (Mammalia: Carnivora: Mustelidae): in Ratnagiri, Maharashtra, India

- Swanand Patil \& Kranti Yardi, Pp. 20511-20516

Wildlife at the crossroads: wild animal road kills due to vehicular collision on a mountainous highway in northwestern Himalayan region

- Muzaffar A. Kichloo, Asha Sohil \& Neeraj Sharma, Pp. 20517-20522

Notes

Robiquetia gracilis (Lindl.) Garay-a new record to the flora of Anamalai Hills, Tami Nadu, India

- B. Subbaiyan, V. Ganesan, P.R. Nimal Kumar \& S. Thangaraj Panneerselvam, Pp. 20523-20525

Ipomoea laxiflora H.J. Chowdhery \& Debta (Convolvulaceae): new records for the Western Ghats and semiarid regions

- Sachin M. Patil, Ajit M. Vasava, Vinay M. Raole \& Kishore S. Rajput, Pp. 20526-20529

Counting the cost: high demand puts Bunium persicum (Boiss.) B.Fedtsch. in jeopardy

- Monika Sharma, Manisha Mathela, Rupali Sharma, Himanshu Bargali, Gurinderjit S Goraya \& Amit Kumar, Pp. 20530-20533

First record of Parasitic Jaeger Stercorarius parasiticus (Aves: Charadriiformes: Stercorariidae) from inland freshwater Inle Lake, Myanmar

- Sai Sein Lin Oo, Myint Kyaw, L.C.K. Yun, Min Zaw Tun, Yar Zar Lay Naung, Soe Naing Aye \& Swen C. Renner, Pp. 20534-20536

\section{Book Review}

Capparis of India

- V. Sampath Kumar, Pp. 20537-20538
Publisher \& Host
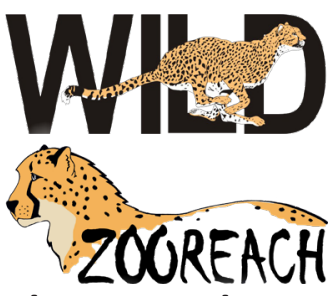

Threatened Taxa 